\title{
Third Trimester Fetal Heart Rate and Doppler Middle Cerebral Artery Blood Flow Velocity Characteristics During Prenatal Selective Serotonin Reuptake Inhibitor Exposure
}

\author{
DAN RURAK, KEN LIM, ARI SANDERS, URSULA BRAIN, WAYNE RIGGS, AND TIM F. OBERLANDER
}

\begin{abstract}
Departments of Obstetrics \& Gynecology [D.R., K.L.] and Pediatrics [U.B., T.F.O.], Faculty of Pharmaceutical Sciences [W.R.], Child \& Family Research Institute [D.R., K.L., A.S., U.B., T.F.O.], University of British Columbia, Vancouver, British Columbia, Canada V6T 1Z1
\end{abstract}

\begin{abstract}
Prenatal selective serotonin reuptake inhibitor (SSRI) exposure increases the risk for adverse neonatal behavioral outcomes; although it is unknown whether altered brain function is present before birth. We investigated fetal vascular and heart rate changes at 36-wk gestation in SSRI-treated women with mood disorders $(n=29)$ [exposed (EXP)] and controls $(n=45)$ [non-EXP (NEXP)]. Fetal middle cerebral artery (MCA) flow parameters and heart rate characteristics were obtained during pre-SSRI dose morning and postdose afternoon sessions. Maternal mood and cord $\mathrm{Hb}$ and hematocrit were measured. Basal fetal heart rate (fHR) did not differ between groups or across the day. The fHR short- and long-term variations, accelerations, and duration of high variability episodes remained lower and did not change across the day in EXP, whereas all increased significantly in NEXP. In both groups, MCA flow velocity and volume flow increased significantly across the day. EXP MCA pulsatility index was significantly lower, as was MCA cross-sectional area. EXP cord $\mathrm{Hb}$ and hematocrit were significantly increased. Prenatal SSRI exposure reduced fetal MCA flow resistance and fHR variability, before and after an SSRI dose, controlling for maternal mood. These changes and the SSRI-related increased red cell indices suggest possible fetal hypoxia. (Pediatr Res 70: 96-101, 2011)
\end{abstract}

Tncreasing use of selective serotonin reuptake inhibitor (SSRI) antidepressants to manage maternal mood disturbance during pregnancy has raised concerns about the longterm effects of increased central serotonergic tone during fetal development (1-3). Widespread observations of neonatal neurobehavioral disturbances $(4,5)$, altered infant stress regulation $(6,7)$, and increased risk for neonatal persistent pulmonary hypertension (8) have been reported. Although persistent pulmonary hypertension in neonates has been supported by findings of increased pulmonary arterial wall thickness, associated with lower oxygen saturation and a higher mortality in fluoxetine-exposed (EXP) newborn rats (9), a subsequent human report did not replicate the earlier human reports (10). Such adverse consequences may reflect the effects of SSRI-related increased levels of the neurotransmitter serotonin during fetal development $(3,11)$. SSRIs primarily act by blocking the

Received October 4, 2010; accepted January 23, 2011

Correspondence: Dan Rurak, Ph.D., Child and Family Research Institute, Room 208C 950 West 28th Avenue, Vancouver BC, Canada V5Z4H4; e-mail: drurak@cw.bc.ca

Supported by the Canadian Institutes of Health Research [T.F.O., CIHR \#MOP 57837]; HELP (Human Early Learning Partnership), Senior Career Award and is the R. Howard Webster Professorship in Child Development (UBC, College of Interdisciplinary Studies) [T.F.O.]; and Investigatorship Award from the Child \& Family Research Institute [D.R.]. serotonin transporter thereby increasing extracellular serotonin levels, and because SSRIs readily cross the placenta and the blood-brain barrier, it is conceivable that they alter early brain development via increased central serotonin levels (12). It remains unclear, however, what mechanisms underlie these disturbances and whether the effects of prenatal SSRI exposure are apparent before birth, long before ongoing antenatal medication exposure ends.

To date, very little is known about SSRI effects on the human fetal physiologic functions (13). In a single report, fetal middle cerebral artery (MCA) Doppler blood flow velocities increased by $18.5 \%$ over an expected clinical level in two fetuses of depressed SSRI-treated pregnant women (14). In pregnant sheep, an 8-d i.v. fluoxetine infusion resulted in initial transient reductions in uterine blood flow and fetal oxygenation, prolonged reduction in fetal rapid eye movement (REM) sleep, and augmented prepartum cortisol increase (1517). Altered fetal REM sleep could affect cerebral blood flow and metabolism via changes between quiet and REM sleep which may in turn have long-term effects on brain growth and maturation (18). It remains unknown whether these fetal effects reflect an acute but transient pharmacological phenomenon or represent sustained alterations in brain development or function (19).

In this study, we examined fetal heart rate (fHR) variability and Doppler blood flow velocity variables in the MCA, umbilical artery, and uterine arteries at 36-wk gestation in depressed women taking SSRI medications and in non-SSRItreated women. To distinguish acute pharmacological effects from long-term sustained outcomes, we examined fetal blood flow and fHR variability measures before a typical morning SSRI dose (trough) and during an afternoon postdose (peak) session. Given, previous reports of neonatal cardiorespiratory and neurobehavioral disturbances in our sheep model after prenatal SSRI exposure (15-17), we expected blunted fetal brain blood flow indices and heart rate variability during late gestation would be present during both trough (predose) and

\footnotetext{
Abbreviations: ANCOVA, analysis of covariance; EXP, exposed; NEXP, nonexposed; fHR, fetal heart rate; HAM-A, Hamilton Rating Scale for Anxiety; HAM-D, Hamilton Rating Scale for Depression; MCA, middle cerebral artery; PI, pulsatility index; REM, rapid eye movement; SSRI, selective serotonin reuptake inhibitor
} 
peak (1-2 h postdose) drug conditions, reflecting sustained fetal effects rather than an acute pharmacological effect.

\section{METHODS}

This longitudinal observational study was approved by the UBC Research Ethics Board and the BC Women's Hospital Research Review Committee. Informed consent was obtained from EXP and NEXP mothers recruited during their second trimester from a Reproductive Mental Health Clinic, community midwife, and family physician clinics in metropolitan Vancouver. All SSRI-treated mothers were started on medication based on clinical need, had a diagnosis of a mood disorder, and were already on antidepressant medications at the time of conception (Table 1).

Inclusion criteria for both groups included singleton pregnancy, confirmed GA, no known fetal anomalies, ability to give informed consent, and lack of substance abuse. Exclusion criteria for both groups included bipolar disorder, exposure to over the counter medications other than vitamins, and significant maternal medical, obstetrical, or fetal conditions. Mothers in the EXP group who were taking other CNS prescription drugs at the time of study were included (Table 1). However, no mothers in this group were taking more than one SSRI.

Maternal mood. Maternal mood was assessed at the time of enrolment (late second trimester) and the fetal study (mid third trimester) using clinician rated measures [Hamilton Rating Scale for Depression (HAM-D) and Anxiety (HAM-A) (20).

Experimental protocol. On the study morning (35-36 wk), subjects in the SSRI-treated group were asked to withhold their morning dose of SSRI. Both groups were to eat and drink normally before arrival and they were all placed in a semirecumbent, left lateral decubitus position throughout the ultrasound examination except when the left uterine artery was assessed (right lateral decubitus). Fetal and placental position and adequacy of amniotic fluid (amniotic fluid index between 5 and 95 percentile) and fetal movements were documented by ultrasound before study onset. The study protocol involved two 2-h sessions, a morning session $(\sim 0815 \mathrm{~h})$ and an afternoon session $(\sim 1230 \mathrm{~h})$. In-between the morning and afternoon sessions, a standard rest break occurred (120 min) during which a standard lunch was provided while mothers were encouraged to get up and move around during the break.

The Doppler vascular study protocol was performed by K.L. who was blinded to medication status and focused on the four arterial vessels. The pulsatility index (PI), mean Doppler flow velocity, vessel diameter/crosssectional area, and volume flow were determined in the MCA and right and left uterine arteries and the PI alone in the umbilical artery. The scans were done using an Aloka ProSound 5500 with a curvilinear 4-7 MHz: probe using B mode, color flow, and pulse wave Doppler where appropriate. All measurements other than vessel diameter were performed five times and the mean value used for the analysis. The vessel diameter was measured to the nearest $0.1 \mathrm{~mm}$ from the inner dimensions (i.e. inner surface to inner surface) at three different locations several millimeters apart, astride where Doppler sampling was performed. This was repeated three times and the mean of the nine measures taken. The protocol took on average $\sim 40 \mathrm{~min}$. Volume flow in the fetal MCA and uterine arteries was calculated as the product of mean Doppler flow velocity times vessel cross-sectional area (calculated from the measurement of vessel diameter) (21).

The fHR variability study then followed the ultrasound protocol and consisted of computerized cardiotocography (Oxford Sonicaid 8002; Oxford Instruments, UK) performed for the next $50 \mathrm{~min}$. Data collected from the Sonicaid system included baseline fHR, accelerations, decelerations, shortand long-term variation, and minutes of high episodes (22).

Table 1. Specific SSRIs (n), prenatal dose (range), and length of prenatal exposure (mean $\pm S D)$

\begin{tabular}{lcc}
\hline $\begin{array}{c}\text { SSRI antidepressants } \\
(n=29)\end{array}$ & $\begin{array}{c}\text { Dose } \\
(\text { mean } \mathrm{mg})\end{array}$ & $\begin{array}{c}\text { Days of prenatal } \\
\text { exposure }\end{array}$ \\
\hline Fluoxetine (4)*† & $37.5 \pm 26.3(10-60)$ & $216 \pm 96$ \\
Paroxetine (3) & $23.3 \pm 5.8(20-30)$ & $268 \pm 17$ \\
Sertraline (4) & $125 \pm 90(25-200)$ & $188 \pm 98$ \\
Venlafaxine (13)* & $158 \pm 56(56-263)$ & $272 \pm 12$ \\
Citalopram (5) $\dagger \dagger$ & $38 \pm 18(10-60)$ & $273 \pm 18$ \\
\hline
\end{tabular}

* One mother was also treated with bupropion.

$\dagger$ One mother was also treated with quetiapine.

\$ One mother was also treated with trazodone.
Neonatal data. Neonatal measures (GA, birth weight, head circumference, and so on) were obtained from the birth examination records. Cord blood $\mathrm{Hb}$ and hematocrit were measured at $\mathrm{BCWH}$.

Data analysis. Comparisons of maternal group characteristics were undertaken using univariate analyses of variance. Categorical variables were compared using $\chi^{2}$. Repeated measures analyses of covariance (ANCOVA) were used to examine time (morning versus afternoon) $\times$ group ]SSRI EXP versus non-EXP (NEXP)] differences in physiological parameters, using measures of prenatal (third trimester) maternal mood as the key covariate in all analyses. Measures of maternal anxious and depressed mood were used in separate models; however, as depressed and anxious symptoms were highly correlated, only outcomes using HAM-D scores as a covariate are reported. We calculated effect sizes (eta squared, $\eta^{2}$ ) to examine the strength of these associations, as the statistical significance of the findings would be affected by sample size and potentially by multiple comparisons. Univariate analysis of variance was used to determine differences in maternal and fetal group characteristics. A $p$ value of $<0.05$ was considered significant. Values are given and the mean \pm SE. All statistical analyses were carried out using SPSS (18.0; http://www.spss.com/).

\section{RESULTS}

Maternal and fetal/neonatal characteristics. With the exception of maternal mood and weight, maternal characteristics did not differ significantly between exposure groups $(p>$ 0.05 ; Table 2). At both the 27- to 28-wk and 36-wk visits, maternal depressed and anxious mood symptoms were significantly higher among the SSRI-treated group. Daily doses of each commonly prescribed SSRI (fluoxetine, paroxetine, sertraline, venlafaxine, and citalopram) were in a typical therapeutic range and the mean duration of therapy spanned most or all of pregnancy (Table 1). The use of alcohol and tobacco was low and did not differ significantly between groups.

Neonatal outcomes were similar between groups, with the exception of lower Apgar scores at 1 min among EXP neonates (Table 3). GA was lower and the percentage delivering at less the 37-wk gestation was significantly higher (13.8 versus $2.1 \%$ ) in the EXP group. Four EXP and one NEXP infant that delivered $<37$ wk were born between 4 and $10 \mathrm{~d}$ (mean $4.8 \pm 1.5 \mathrm{~d}$ ) after the 36 -wk fetal blood flow study. Head circumference was significantly smaller in EXP (34.2 \pm 0.3 versus $35.1 \pm 0.2 \mathrm{~cm}(p=0.002)$; however, the significance disappeared when GA was added to the regression model. For a variety of technical reasons (i.e. infants were outborn and lack of routine cord blood gas analysis), we were able to obtain cord blood for studies of red cell indices in only

Table 2. Maternal characteristics in the nonexposed and exposed groups (means $\pm S E$ )

\begin{tabular}{lcc}
\hline & $\begin{array}{c}\text { Nonexposed } \\
(n=45)\end{array}$ & $\begin{array}{c}\text { Exposed } \\
(n=29)\end{array}$ \\
\hline Maternal age (y) & $34.3 \pm 4.9$. & $33.5 \pm 6.2$ \\
Maternal weight at 36 wk (kg) & $76.1 \pm 10.5$ & $84.1 \pm 12.6^{*}$ \\
Gravidity & $2.1 \pm 0.1$ & $2.2 \pm 0.9$ \\
Parity & $0.4 \pm 0.6$ & $0.4 \pm 0.6$ \\
Alcohol drinks up to 36-wk study (n) & $478 \pm 8.0$ & $6.94 .7 \pm 12.5$ \\
Education (y) & $17.97 \pm 3.45$ & $17.32 \pm 3.9$ \\
Smoking (n) & 1 & 1 \\
GA on fetal study day (wk) & $36.1 \pm 0.16$ & $36.36 \pm 0.17$ \\
Prenatal mood symptoms & & \\
$\quad$ HAM-A score (27 wk) & $7.9 \pm 5.9$ & $12.8 \pm 6.6^{*}$ \\
HAM-D score (27 wk) & $7.2 \pm 6.2$ & $11.3 \pm 6.7^{*}$ \\
HAM-A (36 wk) & $9.1 \pm 6.3$ & $11.6 \pm 4.54^{*}$ \\
HAM-D (36 wk) & $7.65 \pm 5.97$ & $10.56 \pm 4.5^{*}$ \\
\hline
\end{tabular}

$* p<0.05$, for differences between groups. 
Table 3. Birth outcome data in the exposed and nonexposed groups (mean $\pm S D$ ) and by $<$ or $\geq 37$ wk GA at birth

\begin{tabular}{lcc}
\hline & Nonexposed $(n=45)$ & Exposed $(n=29)$ \\
\hline Birth weight $(\mathrm{g})$ & $3534(431)$ & $3326(394)$ \\
Birth length $(\mathrm{cm})$ & $51.8(2.4)$ & $50.7(2.1)$ \\
Head circumference $(\mathrm{cm})$ & $35.1(1.4)$ & $34.1(1.3)^{*}$ \\
Gender of baby $(\mathrm{M} ; \mathrm{F})$ & $24: 24$ & $10: 13$ \\
Apgar score at $1 \mathrm{~min}$ & $8.63(1.0)$ & $7.0(1.7)^{*}$ \\
Apgar score at 5 min & $9.0(.3)$ & $8.9(.5)$ \\
Gestation age at birth (wk) & $40.1(1.0)$ & $39.6(1.1)^{*}$ \\
\hline
\end{tabular}

$* p<0.05$ for differences between exposed and nonexposed groups.

a subgroup of infants. In EXP $(n=10), \mathrm{Hb}$ and hematocrit were significantly higher compared with $\operatorname{NEXP}(n=11)$ $(182 \pm 7.9$ versus $159 \pm 3.8 \mathrm{mg} / \mathrm{dL}$ and $0.528 \pm 0.06$ versus $0.477 \pm 0.03$, respectively), controlling for gestation age, delivery type, and maternal mood at 36 -wk gestation $(F=$ 6.150; $p=0.031 ; \eta^{2}=0.359$ and $F=7.212 ; p=0.021$; $\eta^{2}=0.396$, respectively). Characteristics of neonates (gestational age, birth weight, and head circumference) with cord blood measurements did not differ significantly from those neonates lacking such estimates $(p>0.05)$.

Umbilical and uterine artery flow characteristics. Overall, there was an exposure by time interaction for patterns of umbilical artery blood flow across the day $(F=4.32, p=$ 0.041, $\eta^{2}=0.058$; Table 4). In the NEXP group, umbilical artery PI decreased significantly from morning to afternoon $\left(F=3.89, p=0.055, \eta^{2}=0.085\right)$, whereas in the EXP group, it did not change significantly over the day but was lower than in the NEXP group in the morning (Table 4). No differences in any of the uterine artery flow characteristics examined were noted $(p<0.05)$.

Fetal movements, heart rate, and heart rate variability. Maternally perceived fetal movement counts did not differ between the NEXP and EXP groups, nor across the day $(F=$ $0.315 ; p=0.57 ; \eta^{2}=0.004$; Fig. 1$)$. Basal heart rate at the onset of each study session was similar between groups and no significant differences emerged between morning and afternoon $(p>0.05)$; however, differences in fHR variability indices emerged between groups and across the day. A significant time by exposure group interaction regarding shortterm variations emerged across the day $(F=6.91, p=0.010$, $\eta^{2}=0.086$ ). Namely, little or no change was observed across the day among $\operatorname{EXP}\left(F=0.234, p=0.632, \eta^{2}=0.009\right.$; Fig.

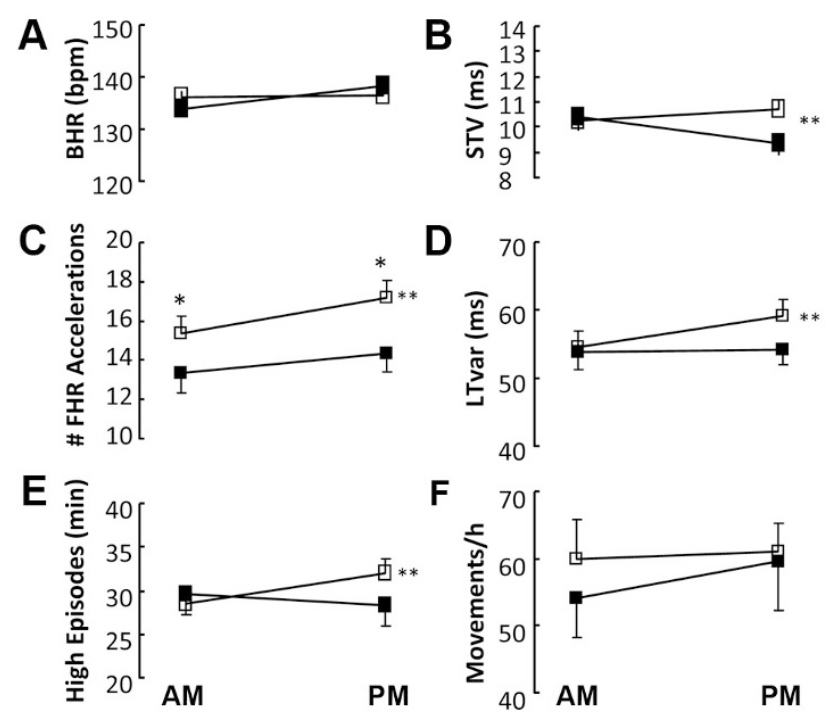

Figure 1. Basal fetal heart rate $(A)$, short-term variability $(B)$, fetal heart rate accelerations $(C)$, long-term variation $(D)$, duration of high variability $(E)$, and fetal movements/h $(F)$ in the nonexposed $(\square)$ and exposed (ם) groups in the morning (AM) and afternoon (PM) monitoring sessions. *ANCOVA $(F=$ $6.91 ; p=0.010 ; \eta^{2}=0.086$ ) for differences between EXP and NEXP groups; **a group by time interaction significantly different from the corresponding $\mathrm{AM}$ value.

1), whereas among NEXP, short-term variation increased significantly from morning to afternoon $(F=9.763, p=$ $\left.0.003, \eta^{2}=0.179\right)$. The number of fHR accelerations in the EXP group were lower compared with NEXP fetuses $(F=$ 6.57, $\left.p=0.012, \eta^{2}=0.083\right)$. Accelerations in EXP remained unchanged from morning to afternoon $(F=0.006, p=0.937$; $\left.\eta^{2}=0.000\right)$, whereas they increased in the $\operatorname{NEXP}(F=3.067$, $p=0.087, \eta^{2}=0.064$; Fig. 2). Similarly, long-term variation in EXP fetuses remained unchanged across the day $(F=$ $\left.0.215 ; p=0.647 ; \eta^{2}=0.008\right)$ and among NEXP fetuses increased from morning to afternoon $(F=10.0 ; p=0.003$; $\eta^{2}=0.183$; Fig. 2). The same pattern appeared for duration of high-HR variability episodes, which remained essentially unchanged in $\operatorname{EXP}\left(F=1.96 ; p=0.173 ; \eta^{2}=0.068\right)$ and increased significantly across the day $\operatorname{NEXP}(F=5.18$; $p=0.028 ; \eta^{2}=0.103$; Fig. 2). Number of decelerations did not differ between groups or over the day $(p>0.05$, data not shown).

Table 4. Umbilical artery pulsatility index (PI) and mean uterine artery (UtA) PI, mean flow velocity (MVC), vessel cross-sectional area, and total uterine artery blood flow in the nonexposed and exposed groups

\begin{tabular}{|c|c|c|c|c|}
\hline \multirow[b]{2}{*}{ Variable } & \multicolumn{2}{|c|}{ Nonexposed $(n=45)$} & \multicolumn{2}{|c|}{ Exposed $(n=28)$} \\
\hline & $\mathrm{AM}$ & PM & $\mathrm{AM}$ & PM \\
\hline Umbilical artery PI & $0.957 \pm 0.022$ & $0.883 \pm 0.021^{*}$ & $0.871 \pm 0.027 \dagger$ & $0.872 \pm 0.029$ \\
\hline UtA PI & $0.672 \pm 0.023 \ddagger$ & $0.691 \pm 0.022$ & $0.671 \pm 0.034 \S$ & $0.684 \pm 0.030$ \\
\hline UtA MVC $(\mathrm{cm} / \mathrm{s})$ & $107.0 \pm 4.8$ & $101.9 \pm 3.9$ & $104.2 \pm 5.3$ & $104.9 \pm 6.1$ \\
\hline UtA area $\left(\mathrm{cm}^{2}\right)$ & $0.172 \pm 0.008$ & $0.171 \pm 0.009$ & $0.166 \pm 0.010$ & $0.166 \pm 0.008$ \\
\hline UtA flow (mL/min) & $2159.4 \pm 102.7$ & $2123.8 \pm 144.2$ & $1934.7 \pm 165.3$ & $2124.1 \pm 176.3$ \\
\hline
\end{tabular}

AM, morning; PM, afternoon.

* ANCOVA, $p<0.05$, for change from the corresponding morning value.

$\dagger$ ANCOVA, $p<0.05$, for differences between groups.

$\ddagger n=21$, only used subjects where we had complete data from morning and afternoon study sessions.

$\S n=32$ for UtA variables. 


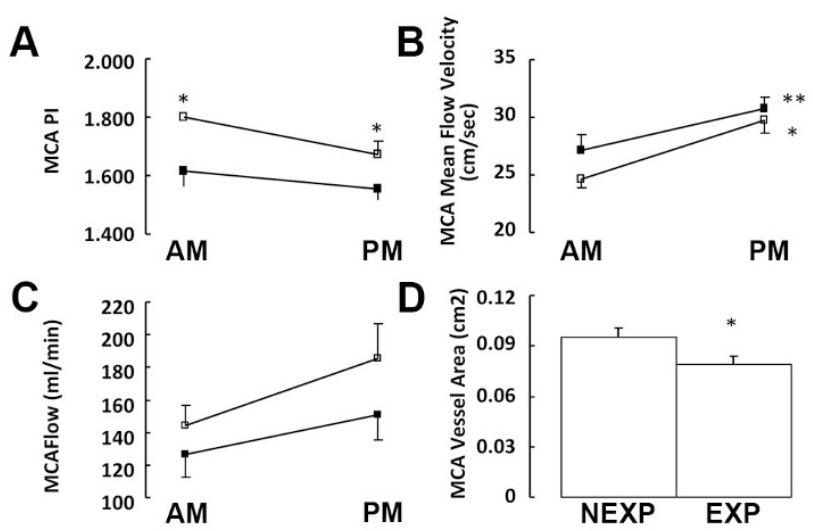

Figure 2. Middle cerebral artery pulsatility index (A), mean blood flow velocity $(B)$, volume flow $(C)$, and vessel cross-sectional area $(D)$ in the NEXP $(\square)$ and EXP $(\square)$ groups in the morning (AM) and afternoon (PM) monitoring sessions. MCA cross-sectional area is represented as a mean, as it was not expected to change with time across the day. $* \operatorname{ANCOVA}\left(F=4.53 ; p=0.037 ; \eta^{2}=0.061\right)$ for differences between EXP and NEXP groups; **a group by time interaction significantly different from the corresponding AM value.

MCA flow. In EXP fetuses, MCA PI was significantly lower during the morning session and remained lower across the day $\left(F=4.53 ; p=0.037 ; \eta^{2}=0.061 ;\right.$ Fig. 2$)$. Changes across the day were not significant in either group $(p>0.05)$. In both groups, MCA mean flow velocity $\left(F=5.43 ; p=0.024 ; \eta^{2}=\right.$ $0.093)$ and MCA volume flow when expressed as a percentage change increased across the day $(p<0.05)$, by $47.0 \pm 13.7 \%$ and $34.0 \pm 15.6 \%$ in the NEXP and EXP groups, respectively. This is consistent with the increase in mean flow velocity (volume flow $=$ mean flow velocity $\times$ cross-sectional area). MCA vessel cross-sectional area (calculated from the vessel diameter) was significantly lower in the EXP compared with the NEXP group, with the difference averaging $17.4 \%(p=$ $0.029)$. MCA vessel diameter was significantly related to birth weight $(p=0.036)$ but not to gestation length or head circumference. However, the association was weak, accounting for only $8 \%$ of the variance in vessel diameter and could not account for the difference between NEXP and EXP. Importantly, all of these outcomes were observed when controlling for maternal depressed (HAM-D) or anxious (HAM-A) mood symptoms at $36 \mathrm{wk}$.

\section{DISCUSSION}

In this study, typical antenatal clinical assessment tools demonstrated that prenatal SSRI exposure was associated with a blunted fHR characteristics and MCA flow impedance both before and after a typical daily dose of the antidepressant, at 36-wk gestation. First, among the EXP fetuses, there were not the increases in fHR variability from morning to afternoon that occurred in the NEXP group. This included measures of short- and long-term variation, heart rate accelerations, and the duration of high variability episodes. Heart rate accelerations in the EXP group were lower over the day compared with the NEXP group. Second, among EXP, MCA PI was lower and remained so across the day. MCA cross-sectional area was also lower compared with NEXP fetuses. In both groups, mean MCA flow velocity and percentage volume flow increased across the day. Third, in the EXP group, there was reduced GA at birth, a higher $\mathrm{Hb}$ and hematocrit, and increased incidence of preterm birth, as reported previously $(23,24)$.

Increased fHR variability across the study day in the NEXP fetuses may reflect a typical diurnal variation in fHR, previously reported in the near-term human fetus (25). The mechanisms involved in this circadian rhythm have not been elucidated in the human, but in pregnant sheep, fetal circadian rhythms in activity, behavior, and cardiovascular function are linked to a fetal plasma melatonin rhythm, which is derived at least in part from the maternal melatonin rhythm via placental transfer $(26,27)$. Why the fHR variables in the EXP group did not show the AM to PM changes remains unclear. Serotonin plays a major role in the entrainment of circadian rhythms (28); however, previously an 8-d i.v. infusion of fluoxetine to pregnant sheep in late gestation did not alter maternal or fetal circadian rhythms (29). It is possible that the much longer duration of SSRI exposure in the human fetuses in the current study could have affected circadian rhythms; assessment of the development of circadian rhythms in newborns EXP in utero to SSRIs may help to clarify this issue. Alternatively, the SSRIs could have interrupted the links between the fetal circadian rhythm generator and the brain stem nuclei that control heart rate.

The number of fHR accelerations were significantly less in the EXP group compared with the NEXP group in both morning and afternoon study sessions, and there were also trends for reduced long-term variation and duration of high variability episodes in the EXP group. These variables are reduced with fetal growth restriction and this has been attributed to delayed maturation of the CNS (30). Although we did not find a significantly lower birth weight in the EXP group, reduced birth weight and increased frequency of growth restriction in SSRI EXP fetuses have been reported $(23,24)$. With increasing growth restriction, fetal biophysical variables including movement and tone are impaired (30). Fetal movements did not differ between groups, however these estimates were based on maternal perceptions of fetal movement, which may underestimate fetal movements (31).

In both groups, decreased MCA PI, increased MCA mean velocity and percentage increase in MCA volume flow were observed, suggestive of increase in MCA perfusion across the day. These changes may reflect circadian rhythms in fetal brain perfusion, similar to a fetal lamb circadian rhythm in carotid arterial blood flow (32). Although changes from morning to afternoon MCA Doppler indices represent circadian rhythms, this suggests that SSRIs do not affect fetal circadian rhythms, but rather affect links between the circadian rhythm generator and entrained physiologic variables, such as fHR variability.

Decreased fetal cerebral arterial vessel resistance indices occur with fetal growth restriction and the accompanying hypoxia in humans and animals (30). Given that MCA PI was significantly lower in the EXP group, this could represent a redistribution of cardiac output to maintain the delivery of oxygen and nutrient molecules to vital organs such as the heart, brain, and adrenal gland by increasing blood flow (33, 34). In EXP fetuses, decreased MCA PI was not accompanied 
by increased volume flow, and this may have been secondary to a smaller cross-sectional MCA area. Because growth of the vascular system seems to be linked to the metabolism of the tissues perfused (35), our findings may suggest either a reduced metabolic rate and/or reduced volume of the brain tissue perfused by the MCA in the EXP group.

Reduced number of fHR accelerations and lower MCA PI may also reflect fetal hypoxemia, as do increased cord blood $\mathrm{Hb}$ and hematocrit in EXP fetuses. Intrauterine hypoxia is a stimulus for increased fetal erythropoietin and circulating nucleated red cells, subsequently leading to long-term increased $\mathrm{Hb}$ and hematocrit (36). If prenatal SSRI exposure is indeed associated with fetal hypoxemia, then it is of a longterm nature. In an animal model, i.v. fluoxetine administration to pregnant ewes resulted in transient fetal hypoxemia and reduced uterine blood flow (15), reflecting a transient increase in blood serotonin (37) and serotonin's action as a potent uterine vasoconstrictor (38).

Overall, our findings raise the possibility that SSRIs adversely affect the fetus via at least two mechanisms. First, there could be a direct central effect of altered serotonergic tone that influences brain development and maturation, leading to changes in brain blood flow, lowered metabolic rate or tissue volume in the brain region perfused by the MCA, and altered prenatal cardiac autonomic control. During brain development, serotonin acts as a regulatory factor influencing cell proliferation, migration, and differentiation. Thus, SSRIelicited alterations in brain serotonin levels could affect these processes; hence, prenatal SSRI exposure in rats alters cell proliferation and survival in multiple areas of the fetal brain (39). Second, there could be systemic effects of transient increases in blood serotonin associated with each daily SSRI dose, resulting in repetitive bouts of reduced uterine blood flow and fetal hypoxemia, which could lead to a redistribution of fetal cardiac output as well as endocrine and metabolic changes affecting fetal growth, brain development, and gestation length $(38,40)$.

A number of key limitations need mentioning. Although there are no reasons to believe that the individual SSRI medications may have distinguishable effects, our cohort was too small to detect differences in the effects of the individual SSRIs. Furthermore, some mothers were also treated, although for short periods, with other psychotropic medications, we could not distinguish an effect specific to these medications. Although four of EXP fetuses delivered $<37.0 \mathrm{wk}$, they did so a mean of $5.0 \pm 1.9 \mathrm{~d}$ after our blood flow studies, thus it is unlikely that we observed the early signs of a preterm labor. Importantly, comparing blood flow differences between fetuses who went on to deliver at term, compared with those that delivered preterm, indicated no differences between these groups and thus the potential effects of a preterm labor were an unlikely explanation for our findings. Furthermore, we assumed that mood differences and SSRI exposure were the main independent variables, and randomization between exposure groups was not possible. Thus, not accounting for unmeasured maternal illness characteristics and related physiological alterations inherent to perinatal maternal mood disorders may have contributed to an ascertainment bias. How- ever, if SSRIs are effective in treating these mood disorders, they should lessen the negative impacts on the offspring, whereas in our population-based study of neonatal outcomes after prenatal SSRI exposure indicated that maternal depression and SSRIs have additive negative effects (24).

In summary, fetal SSRI exposure reduced fetal MCA flow resistance and fHR variability and decreased MCA crosssectional area both before and after a typical prenatal maternal SSRI dose, controlling for antenatal maternal mood. Moreover, prenatal SSRI exposure was associated with increased cord blood $\mathrm{Hb}$ and hematocrit. These findings may reflect an SSRI-related pharmacologic, vascular, or metabolic influences; however, exposure-related increase in $\mathrm{Hb}$ and hematocrit suggest possible fetal hypoxia and sustained in utero SSRI related changes in fetal brain blood flow and functional effects. Further studies are needed to examine the mechanisms that link fetal SSRI exposure with altered central blood flow and behavioral outcomes in the newborn period.

Acknowledgments. We thank the mothers and their infants who participated and contributed to this work, as well as Mary Beckingham, Kiran Greywall, and Wendy Frasca for their contributions in organizing and facilitating this research program. We thank Shelley Soanes and Bruce Duncan for their assistance during the study sessions. We also acknowledge the contribution of our statistical consultant, Dr. Michael Papsdorf.

\section{REFERENCES}

1. Shadigian E, Bauer ST 2005 Pregnancy-associated death: a qualitative systematic review of homicide and suicide. Obstet Gynecol Surv 60:183-190

2. Kinsella MT, Monk C 2009 Impact of maternal stress, depression and anxiety on fetal neurobehavioral development. Clin Obstet Gynecol 52:425-440

3. Oberlander TF, Gingrich JA, Ansorge MS 2009 Sustained neurobehavioral effects of exposure to SSRI antidepressants during development: molecular to clinical evidence. Clin Pharmacol Ther 86:672-677

4. Simon GE, Cunningham ML, Davis RL 2002 Outcomes of prenatal antidepressant exposure. Am J Psychiatry 159:2055-2061

5. Moses-Kolko EL, Bogen D, Perel J, Bregar A, Uhl K, Levin B, Wisner KL 2005 Neonatal signs after late in utero exposure to serotonin reuptake inhibitors: literature review and implications for clinical applications. JAMA 293:2372-2383

6. Oberlander TF, Eckstein GR, Fitzgerald C, Ellwood AL, Misri S, Rurak D, Riggs KW 2002 Prolonged prenatal psychotropic medication exposure alters neonatal acute pain response. Pediatr Res 51:443-453

7. Zeskind PS, Stephens LE 2004 Maternal selective serotonin reuptake inhibitor use during pregnancy and newborn neurobehavior. Pediatrics 113:368-375

8. Chambers CD, Hernandez-Diaz S, Van Marter LJ, Werler MM, Louik C, Jones KL, Mitchell AA 2006 Selective serotonin-reuptake inhibitors and risk of persistent pulmonary hypertension of the newborn. N Engl J Med 354:579-587

9. Fornaro E, Li D, Pan J, Belik J 2007 Prenatal exposure to fluoxetine induces fetal pulmonary hypertension in the rat. Am J Respir Crit Care Med 176:1035-1040

10. Andrade SE, McPhillips H, Loren D, Raebel MA, Lane K, Livingston J, Boudreau DM, Smith DH, Davis RL, Willy ME, Platt R 2009 Antidepressant medication use and risk of persistent pulmonary hypertension of the newborn. Pharmacoepidemiol Drug Saf 18:246-252

11. Wichman CL, Fothergill A, Moore KM, Lang TR, Heise RH Jr, Watson WJ 2008 Recent trends in selective serotonin reuptake inhibitor use in pregnancy. J Clin Psychopharmacol 28:714-716

12. Laine K, Heikkinen T, Ekblad U, Kero P 2003 Effects of exposure to selective serotonin reuptake inhibitors during pregnancy on serotonergic symptoms in newborns and cord blood monoamine and prolactin concentrations. Arch Gen Psychiatry 60:720-726

13. Richardson BS 1992 The effect of behavioral state on fetal metabolism and blood flow circulation. Semin Perinatol 16:227-233

14. Emory EK, Dieter JN 2006 Maternal depression and psychotropic medication effects on the human fetus. Ann N Y Acad Sci 1094:287-291

15. Morrison JL, Chien C, Gruber N, Rurak D, Riggs W 2001 Fetal behavioural state changes following maternal fluoxetine infusion in sheep. Brain Res Dev Brain Res 131:47-56

16. Morrison JL, Chien C, Riggs KW, Gruber N, Rurak D 2002 Effect of maternal fluoxetine administration on uterine blood flow, fetal blood gas status, and growth. Pediatr Res 51:433-442 
17. Morrison JL, Riggs KW, Chien C, Gruber N, McMillen IC, Rurak DW 2004 Chronic maternal fluoxetine infusion in pregnant sheep: effects on the maternal and fetal hypothalamic-pituitary-adrenal axes. Pediatr Res 56:40-46

18. Richardson BS, Patrick JE, Abduljabbar H 1985 Cerebral oxidative metabolism in the fetal lamb: relationship to electrocortical state. Am J Obstet Gynecol 153:426431

19. Oberlander TF, Papsdorf M, Brain UM, Misri S, Ross C, Grunau RE 2010 Prenatal effects of selective serotonin reuptake inhibitor antidepressants, serotonin transporter promoter genotype (SLC6A4), and maternal mood on child behavior at 3 years of age. Arch Pediatr Adolesc Med 164:444-451

20. Hamilton M 1959 The assessment of anxiety states by rating. Br J Med Psychol 32:50-55

21. Erskine RL, Ritchie JW 1985 Quantitative measurement of fetal blood flow using Doppler ultrasound. Br J Obstet Gynaecol 92:600-604

22. Dawes GS, Moulden M, Redman CW 1996 Improvements in computerized fetal heart rate analysis antepartum. J Perinat Med 24:25-36

23. Chambers CD, Johnson KA, Dick LM, Felix RJ, Jones KL 1996 Birth outcomes in pregnant women taking fluoxetine. N Engl J Med 335:1010-1015

24. Oberlander TF, Warburton W, Misri S, Aghajanian J, Hertzman C 2006 Neonata outcomes after prenatal exposure to selective serotonin reuptake inhibitor antidepressants and maternal depression using population-based linked health data. Arch Gen Psychiatry 63:898-906

25. Visser GH, Goodman JD, Levine DH, Dawes GS 1982 Diurnal and other cyclic variations in human fetal heart rate near term. Am J Obstet Gynecol 142.535-544

26. Zemdegs IZ, McMillen IC, Walker DW, Thorburn GD, Nowak R 1988 Diurna rhythms in plasma melatonin concentrations in the fetal sheep and pregnant ewe during late gestation. Endocrinology 123:284-289

27. Houghton DC, Walker DW, Young IR, McMillen IC 1993 Melatonin and the light-dark cycle separately influence daily behavioral and hormonal rhythms in the pregnant ewe and sheep fetus. Endocrinology 133:90-98

28. Morin LP 1999 Serotonin and the regulation of mammalian circadian rhythmicity. Ann Med 31:12-33
29. Morrison JL, Rurak DW, Chien C, Kennaway DJ, Gruber N, McMillen IC, Riggs KW 2005 Maternal fluoxetine infusion does not alter fetal endocrine and biophysical circadian rhythms in pregnant sheep. J Soc Gynecol Investig 12:356-364

30. Baschat AA 2004 Fetal responses to placental insufficiency: an update. BJOG 111:1031-1041

31. Hijazi ZR, East CE 2009 Factors affecting maternal perception of fetal movement. Obstet Gynecol Surv 64:489-497

32. Jensen EC, Bennet L, Guild SJ, Booth LC, Stewart J, Gunn AJ 2009 The role of the neural sympathetic and parasympathetic systems in diurnal and sleep state-related cardiovascular rhythms in the late-gestation ovine fetus. Am J Physiol Regul Integr Comp Physiol 297:R998-R1008

33. Gagnon R, Lamb T, Richardson B 1997 Cerebral circulatory responses of near-term ovine fetuses during sustained fetal placental embolization. Am J Physiol 273:H2001-H2008

34. Richardson BS, Bocking AD 1998 Metabolic and circulatory adaptations to chronic hypoxia in the fetus. Comp Biochem Physiol A Mol Integr Physiol 119:717-723

35. Adair TH, Gay WJ, Montani JP 1990 Growth regulation of the vascular system: evidence for a metabolic hypothesis. Am J Physiol 259:R393-R404

36. Teramo KA, Widness JA 2009 Increased fetal plasma and amniotic fluid erythropoietin concentrations: markers of intrauterine hypoxia. Neonatology 95:105-116

37. Ortiz J, Artigas F 1992 Effects of monoamine uptake inhibitors on extracellular and platelet 5-hydroxytryptamine in rat blood: different effects of clomipramine and fluoxetine. Br J Pharmacol 105:941-946

38. Morrison JL 2008 Sheep models of intrauterine growth restriction: fetal adaptations and consequences. Clin Exp Pharmacol Physiol 35:730-743

39. Hodes GE, Hill-Smith TE, Suckow RF, Cooper TB, Lucki I 2010 Sex-specific effects of chronic fluoxetine treatment on neuroplasticity and pharmacokinetics in mice. J Pharmacol Exp Ther 332:266-273

40. Challis JRG, Matthews SG, Gibb W, Lye SJ 2000 Endocrine and paracrine regulation of birth at term and preterm. Endocr Rev 21:514-550 